\title{
Left Posteromedial Middle Peripheral Zone of Prostate
}

National Cancer Institute

\section{Source}

National Cancer Institute. Left Posteromedial Middle Peripheral Zone of Prostate. NCI

Thesaurus. Code C128602.

The region of the prostate that is located on the anatomical left side of the posteromedial portion of the middle division of the peripheral zone. 